\title{
A C-level flare observed in an arch filament system: reconnection between pre-existing and emerging field lines?
}

\author{
F. Zuccarello ${ }^{1}$, V. Battiato ${ }^{2}$, L. Contarino ${ }^{2}$, S. Guglielmino ${ }^{1}$, P. Romano ${ }^{2}$, and D. Spadaro ${ }^{2}$ \\ 1 Dipartimento di Fisica e Astronomia - Sezione Astrofisica, Università di Catania, via S. Sofia 78, 95123 Catania, Italy \\ e-mail: fzu@oact.inaf.it \\ 2 INAF - Osservatorio Astrofisico di Catania, via S. Sofia 78, 95123 Catania, Italy
}

Received 30 November 2007 / Accepted 25 June 2008

\section{ABSTRACT}

\begin{abstract}
Context. Observations show that solar flares are often caused by the emergence of new magnetic flux from subphotospheric layers and by the interaction of the rising field lines with the ambient magnetic field. In this framework, recent theoretical models of magnetic flux emergence have investigated the effectiveness of magnetic reconnection as a function of the old and new flux system relative orientations.

Aims. We aim to compare phenomena that occurred in an active region, before and during a small flare, with the effects of magnetic reconnection between nearly parallel magnetic field lines, foreseen by these models.

Methods. We analyzed high resolution photospheric and chromospheric data acquired during a coordinated observational campaign performed with the THEMIS telescope in IPM mode, as well as MDI magnetograms and TRACE 1600 and $171 \AA ̊$ images, to investigate the dynamics and the magnetic configuration of the active region hosting the flare.

Results. An emerging arch filament system (AFS) was observed in the area between the two main sunspots: it showed typical upward motion at the arch tops and plasma downward motion at the footpoints. A C-level flare, characterized by a factor of 3 peak enhancement in the GOES X-ray emission with respect to the pre-event background, occurred in this zone, where the configuration of the emerging magnetic field lines showed a small $\left(\sim 12^{\circ}\right)$ relative inclination with respect to the old flux system.

Conclusions. In an active region (age $\geq 6$ days) a new magnetic flux bundle emerged between the two main polarity spots. It gave rise to the formation of pores in the photosphere and to an AFS in the chromosphere. The interaction between the new and the pre-existing field lines, characterized by a small relative inclination, might have caused a weak reconnection process and given rise to the C-level flare. This result is in broad agreement with numerical simulations predicting very limited reconnection when the two flux systems have an almost parallel orientation.
\end{abstract}

Key words. Sun: activity - Sun: flares - Sun: magnetic fields

\section{Introduction}

The formation of active regions in the solar atmosphere is a complex phenomenon that involves several processes, initially including the dynamo mechanism of re-generation of the toroidal field at the basis of the convection zone and later on the buoyancy and rising of magnetic flux tubes from the subphotospheric layers, and finally the interaction of these flux tubes with the low density plasma of the solar atmosphere and with any pre-existing magnetic fields.

Recently, the possibility of performing high resolution observations in the optical and EUV ranges has given us the possibility to investigate the details of magnetic flux emergence in the solar atmospheric layers and to obtain new information on how the emerging flux tubes interact with the ambient plasma and with pre-existing magnetic fields.

In previous papers (Spadaro et al. 2004; Zuccarello et al. 2005), using high resolution observations performed with the THEMIS telescope, we have confirmed that in both long-lived (i.e., lifetime $\geq 14$ days) and short-lived active regions, these phases are characterized by the presence of arch-shaped features, named arch filament system (AFS), that show an upward motion at their tops and plasma downward motion along their legs.

The AFS arches can be identified with the several strands in which emerging flux tubes, rising from the high plasma $\beta$ internal layers, are fragmented during their ascent, and their dynamical behaviour has been interpreted as indicating the upward motion of the flux tubes and simultaneous plasma leakage from their footpoints.

Another characteristic that has been observed is that both upward and downward motion decreases over time, indicating that the rising arches are decelerated as they meet ambient conditions characterized by lower density and by magnetic domains already established in these layers, while the arch expansion may cause a decrease of the plasma downflow.

However, this picture is very simplified, as it does not take into account the observed asymmetries between the preceding and the following legs, as well as other phenomena taking place during active region formation (magnetic reconnection between old and new flux systems, thermal energy budget, magnetic coalescence, presence of shock waves, etc.).

In particular, we want to concentrate on a particular aspect of active region formation related to the observational evidence that magnetic flux emergence is not a process occurring in one single and abrupt step. Rather, it is often observed that it takes place in several, different and discontinuous steps: for instance, it is not rare to observe the sudden appearance and formation of new pores and sunspots in an already developed and apparently stable active region. 
Table 1. THEMIS fields of view.

\begin{tabular}{cccccc}
\hline \hline Field of view & Description & Lat & Long & Observing time (UT) & Performed sequences \\
\hline 1 & following sunspot & $09.4 \mathrm{~S}$ & $16.7 \mathrm{~W}$ & $10: 51-11: 45$ & 30 \\
2 & following sunspot & $08.9 \mathrm{~S}$ & $16.8 \mathrm{~W}$ & $10: 52-11: 44$ & 28 \\
3 & pores & $08.8 \mathrm{~S}$ & $18.1 \mathrm{~W}$ & $12: 38-13: 11$ & 24 \\
4 & pores & $09.2 \mathrm{~S}$ & $17.7 \mathrm{~W}$ & $14: 47-16: 11$ & 59 \\
5 & preceding sunspot & $09.0 \mathrm{~S}$ & $18.6 \mathrm{~W}$ & $17: 04-18: 50$ & 73 \\
\hline
\end{tabular}

This step-by-step process implies interaction of the newly emerging flux tube bundle with the old one and this situation can easily give rise to rearrangements of the magnetic field configuration by means of magnetic reconnection and, eventually, to flares. Observations show that the process of flux emergence in the corona often results in interactions that give rise to local brightenings observed in all wavelengths, from white light to X-ray, passing through EUV lines, as seen for instance in TRACE images.

The problem of the emergence of new magnetic flux systems in a volume occupied by a pre-existing coronal magnetic field has been investigated by Longcope et al. (2005), who showed how the new flux regions became connected to the coronal field using simple flux estimates for the flaring structures. They noticed that reconnection was not active for a long initial phase (1 day), after which a large amount of the newly emerging flux connected to the coronal field on a relatively short timescale (3-6 h).

In this context, Galsgaard et al. (2007) studied how the interactions depend on the structure of the two initially unconnected magnetic flux systems. Due to the present inability to directly track magnetic field lines from existing observational data, only numerical simulations allow us to control the effects of the interaction in the environment into which flux emergence occurs. However, because the timescale involved in the experiments only covers $20 \mathrm{~min}$, while in the observations the evolution is of the order of one day, it is impossible to make a real comparison, but it is interesting to compare the structures arising from the numerical simulations with those from observations.

The fundamental parameter in the above analysis is the orientation between the two flux systems. Galsgaard et al. (2007) found a smooth increase over time in the flux interaction, and effective reconnection which results in high-velocity $\left(\sim 200 \mathrm{~km} \mathrm{~s}^{-1}\right)$ outflows ejected sideways from the reconnection sites, most efficient when the two flux systems are antiparallel, while when the relative orientation is not favorable, i.e. the two flux systems are parallel, there is no efficient reconnection and the coronal field is pushed upwards forming a hill-shaped interface. Only at a later stage of the evolution do some ambient field lines slide down along the slope of the emerging flux, reconnecting at lower heights, closer to the photosphere, without the plasma heating observed by, e.g., TRACE. They also noticed that the bipolar patterns of the initial flux tube are maintained, even for a height of only $\sim 3500 \mathrm{~km}$ above the photosphere, and that the excess magnetic pressure of the subphotospheric magnetic field expels the flux upwards, opening the volume for a new bipolar structure. Moreover, it was inferred that the fate of the new coronal magnetic field, i.e. its length and structure, strongly depends on the total amount of emerging flux, on the amount of coronal ambient flux and their ability to reconnect with each other.

In this paper we describe a C-level flare that occurred in an active region after the appearance, between the two main spots, of new pores at the photopheric level and of an AFS at the chromospheric level. We show that the flare may be interpreted as the signature of magnetic rearrangement and reconnection between the pre-existing magnetic field lines and the newly emerging ones: both flux systems show a similar orientation, indicating that the magnetic field topology might be considered as an example of interaction of two nearly parallel fields.

\section{Data description}

We observed the Active Region NOAA 10401 on July 13, 2003, using data acquired by ground-based (THEMIS/IPM) and satellite instruments (SOHO/MDI, TRACE).

High resolution images were acquired using the Télescope Héliographique pour l'Étude du Magnétisme et des Instabilités Solaires (THEMIS) operating in IPM mode. These images were acquired in 18 spectral points, 11 along the profile of the $\mathrm{H}_{\alpha}$ line $(\lambda=6562.808 \AA,+/-0.25 \AA,+/-0.50 \AA,+/-0.75 \AA,+/-1.00 \AA$, $+/-1.50 \AA$, with an exposure time of $300 \mathrm{~ms}$ ) and 7 along the Fe I line $(\lambda=5575.960 \AA)$. At the same time images in broad band centered at $5380.960 \AA$ were acquired. The period employed to acquire the images in the 18 spectral points was $\sim 1$ min. The temporal resolution between two spectral images is $\sim 3 \mathrm{~s}$ and the spatial resolution ranges from $\sim 0.2$ to $\sim 0.4$ arcsec. During the observing run we took images in five different fields of view, in order to scan the whole area of the active region. The analyzed dataset is reported in Table 1.

We also used full disk line of sight magnetograms acquired by MDI/SOHO, as well as 171 and $1600 \AA$ high resolution images taken by TRACE to study the magnetic and morphological evolution of NOAA 10401. Data on the flare activity of NOAA 10401 during its passage across the solar disk were obtained from the NOAA Active Region Reports.

The MDI full disk magnetograms used were taken from 00:02 UT to 22:46 UT on July 13, with an average time cadence of $96 \mathrm{~min}$ and a spatial resolution of 2 arcsec.

TRACE images were taken in full resolution mode, i.e. $768 \times 768$ pixels, with a pixel size of 0.5 arcsec and a field of view of $6.4 \times 6.4 \mathrm{arcmin}$; the time cadence was $\sim 30 \mathrm{~s}$. TRACE observed NOAA 10401 for several days, but we used only the images acquired on July 13, during the time interval between 11:12 and 12:59 UT, when a flare of class C1.2 was observed.

\section{NOAA 10401 global morphology and evolution}

NOAA 10401 appeared on the Eastern limb on July 06, 2003, with an average latitude of $9 \mathrm{~S}$, it increased its area until July 11, and disappeared on the Western limb on July 17.

Figure 1 shows the active region in photosphere (WL) on July 13, as observed by TRACE in white light: it shows that the morphology of NOAA 10401 is characterized by two main spots and some pores (it has a $\beta$ configuration in the Mt. Wilson classification).

Figure 2 reports two reconstructions of NOAA 10401 obtained from the observations carried out with THEMIS on 


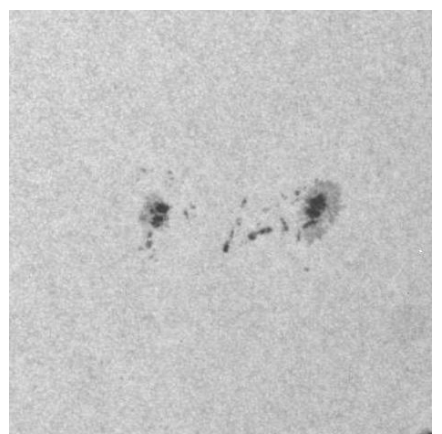

Fig. 1. WL image of NOAA 10401 acquired on July 13 by TRACE at 11:24 UT (fov $\sim 163 \times 163 \mathrm{Mm}^{2}$ ). In this and in the following images, if not specified otherwise, North is on the top, West on the right.

July 13. In particular, Fig. 2a shows the five photospheric fields of view, while Fig. $2 \mathrm{~b}$ reports the corresponding chromospheric fields of view. Details about THEMIS fields of view are reported in Table 1. The fields of view 3 and 4 indicate the presence of an AFS (Fig. 2b), characterized by two main arches in absorption, several faint dark arches and two main, approximately parallel, bright regions corresponding to the AFS footpoints.

\section{The event evolution}

The solar X-ray flux recorded by GOES-10 in the 1-8 $\AA$ channel (see Fig. 3, red line) indicates that on July 13 a C-level flare occurred, beginning at 12:15 UT, peaking at 12:26 UT and ending at 12:46 UT. The peak flux represents an enhancement of a factor of about 3 above the pre-event flux (background) of discintegrated X-rays. Imaging instruments indicated that the flare energy release occurred within NOAA 10401. In Fig. 3 we also report the light curves obtained by TRACE images acquired at $171 \AA$ (dotted line) and $1600 \AA$ (continuous line), respectively. If we compare the emission curves of EUV and X channels, we notice that maxima are shifted by $\sim 6$ min between the EUV and the $1-8 \AA$ channels.

From the sequence of images acquired by TRACE at $1600 \AA$ and $171 \AA$, we inferred the evolution of the flare in the coronal region corresponding to the chromospheric area characterized by the presence of the AFS.

In particular, from the analysis of images at $1600 \AA$, we deduced an increase of brightness in the regions corresponding to the footpoints of the chromospheric AFS, starting at 12:09 UT. The maximum phase is registered at 12:21 UT, when the increased brightness clearly indicates the presence of two, almost parallel, ribbons (see Fig. 4a); the end of the flare is registered at this wavelength at 12:43 UT.

The analysis of images at $171 \AA$ indicates that initially the EUV filament channel corresponding to the most southern AFS arch is dark in the central part and bright at the footpoints. Later on there is a progressive enhancement of emission in the footpoints of the EUV filament channel (12:13 UT), followed by brightenings of arch shaped structures nearly parallel to the main AFS arch during the maximum phase (12:21 UT) (see Fig. 4b); at this time, the presence of the ribbons is evident, even if their brightness at this wavelength is lower than that observed at $1600 \AA$ (compare Fig. 4a with Fig. 4b). At 12:28 UT the brightening propagates at nearby arcades. Later, at 12:53 UT, the brightness disappears.

We also studied the evolution of the small flare at chromospheric level using the THEMIS images relevant to fields of view 3 and 4. In particular, the images relevant to field of view 3 were acquired during the time interval 12:38-13:11 UT, i.e. after the flare peak, but during the flare main phase. These images show an increased brightness in the region corresponding to the western footpoints of the AFS arches. This brightness appears to be rather diffuse and no significant morphological evolution is observed during the time interval analyzed.

In order to determine the values of velocity along the line of sight (los) in the chromosphere (through the $\mathrm{H}_{\alpha}$ line), we considered the Doppler shift of the centroid of the line profile at each spatial point with respect to the median of the centroid in the whole field of view. This method is described in detail in Spadaro et al. (2000) and Zuccarello et al. (2005).

In Fig. 5 we report the field of view 3 at four different times, showing the $\mathrm{H}_{\alpha}$ images (on the left) and the corresponding velocity fields (on the right). This analysis indicates that the region characterized by the brightness increase shows downward plasma motion, reaching values of $\sim 20 \mathrm{~km} \mathrm{~s}^{-1}$ during the time interval 12:38-12:43 UT. During the same time interval the surrounding regions are darker and characterized by upward motion $\left(\sim 3 \mathrm{~km} \mathrm{~s}^{-1}\right)$.

The images relevant to the field of view 4, reported in Fig. 6, were acquired during the time interval 14:47-16:11 UT, i.e., after the end of the flare. These images show the AFS formed between the two main spots, characterized by opposite polarities. The presence of the AFS indicates that on July 13 a new flux tube bundle emerged between the two sunspots. This AFS was characterized by two main dark arches and by a number of smaller dark arches, some of which appeared during the observing run in the central part of the AFS. The brightness of the AFS footpoints is evident in all the images, and indicates the heating of the plasma in these regions. Moreover, the Doppler analysis indicates that the AFS tops are rising in the solar atmosphere ( $\max$ upward velocity $\sim 3 \mathrm{~km} \mathrm{~s}^{-1}$ ), while the plasma is downflowing at the extremities $\left(\sim 10 \mathrm{~km} \mathrm{~s}^{-1}\right)$ (see Fig. 6). This dynamical behaviour is in agreement with previous observations (Spadaro et al. 2004; Zuccarello et al. 2005) and it represents further evidence of the rising of new flux tubes in this area.

The orientation of the AFS arches in the $\mathrm{H} \alpha$ images (see Fig. 6) allow us to conclude that the bundles of field lines forming the AFS are parallel to each other, therefore there is no sign of a twist in the emerging flux tube. Previous studies on AFS morphology and dynamics have shown that the twisting of the newly emerged flux tube is visible in $\mathrm{H} \alpha$ images as AFS arches crossing each other (see, e.g., Strous \& Zwaan 1999, Fig. 6; Spadaro et al. 2004, Fig. 10). Moreover, the absence of clear signatures of twisting of the field lines can also be inferred by the parallel orientation of the small loops indicated by the arrow in Fig. 8d.

\section{Magnetograms analysis}

In order to further investigate the emergence of magnetic flux tubes in the AFS area we analyzed the magnetic evolution of the active region on July 13 from 00:00 UT to 22:24 UT.

As shown in Fig. 7a, on July 13, 2007, at 00:02 UT NOAA 10401 was characterized by a bipolar configuration in the East-West direction. During the hours before the C-level event we observed a significant emergence of new magnetic flux in the region between the two main flux concentrations (Fig. 7b).

We aligned the single magnetograms taken at 00:02 UT and at 12:14 UT using the cross correlation method, to derive a difference map. Using the contours at a chosen level of difference of $2.6 \times 10^{18} \mathrm{Mx} /$ pixel to show the areas characterized by 


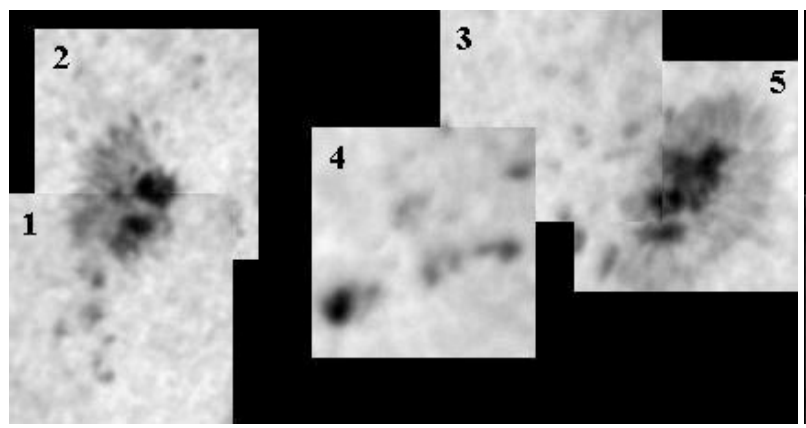

(a)

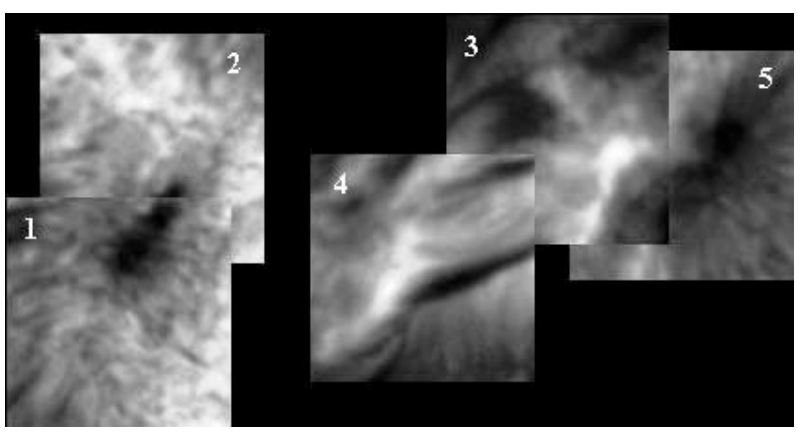

(b)

Fig. 2. Reconstructions of NOAA 10401 obtained from the images acquired by the THEMIS telescope a) in the continuum at $5380.960 \AA$ and $\mathbf{b}$ ) in the centre of the $\mathrm{H}_{\alpha}$ line, on July 13. The numbers in each square indicate the 5 different fields of view scanned by THEMIS (see Table 1). The fov of both images is $\sim 84 \times 43 \mathrm{Mm}^{2}$. The 5 fields of view were acquired at: (1) 10:53 UT, (2) 10:52 UT, (3) 12:38 UT, (4) 14:48 UT, (5) 17:04 UT.
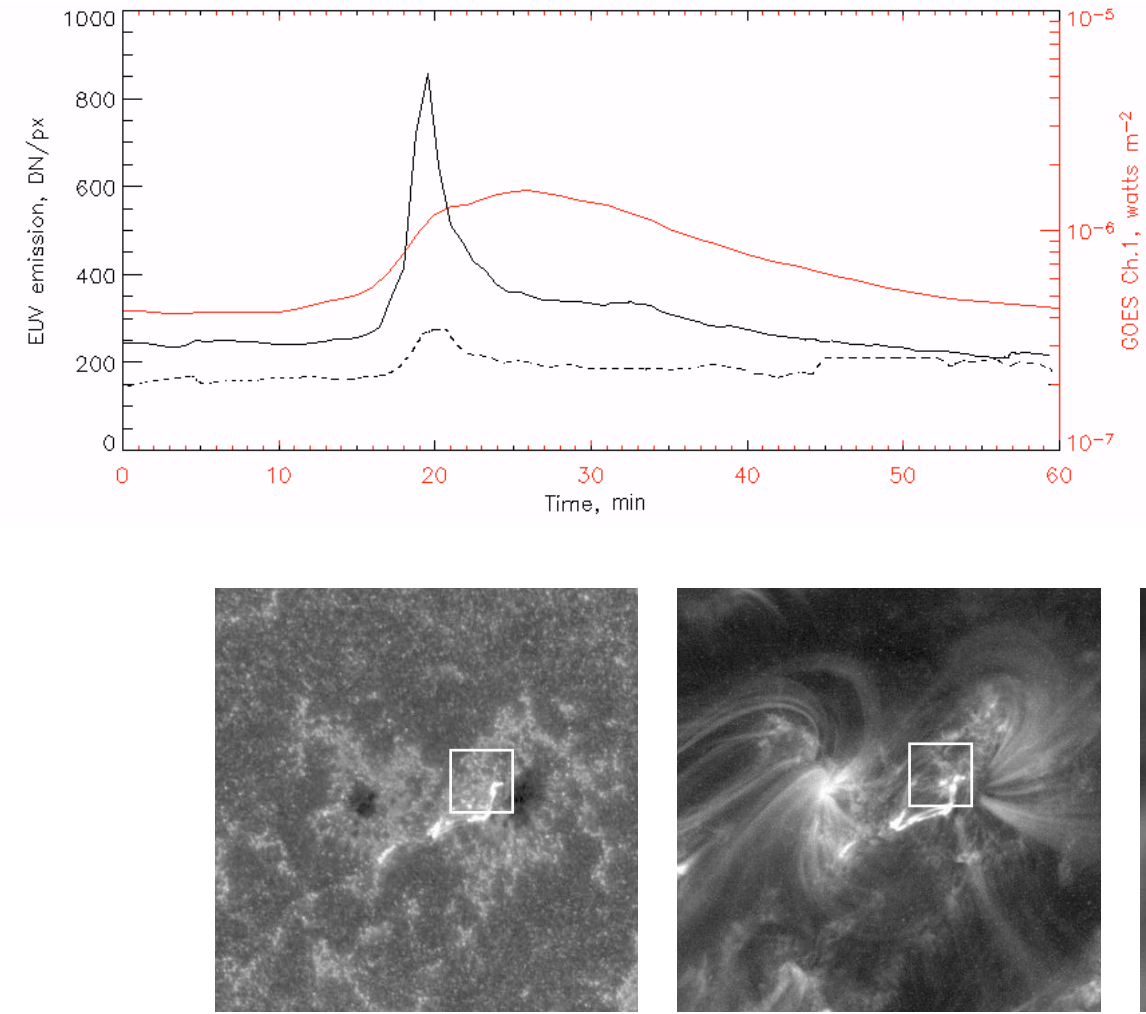

(a)

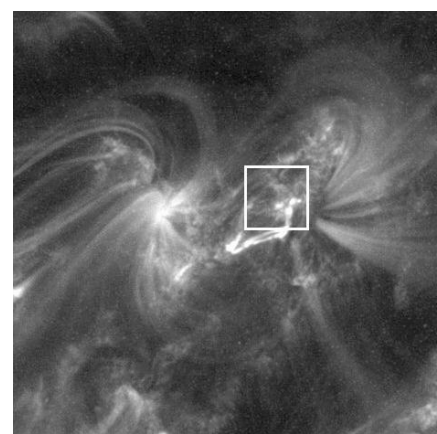

(b)
Fig. 3. Solar X-ray flux recorded by GOES-10 on July 13 from 12:00 UT to 13:00 UT, integrated in the wavelength range $1-8 \AA$ (red line); light curves obtained from TRACE images acquired at $171 \AA$ (dotted line) and at $1600 \AA$ (continuous line).

Fig. 4. Images of NOAA 10401 acquired on July 13 at 12:21 UT by TRACE at $1600 \AA$ a) and $171 \AA$ b). The white rectangles indicate the THEMIS field of view 3, shown in panel c). The fov of a) and b) panels is the same of the MDI magnetograms in Fig. 7.

the most consistent flux emergence we can see in Fig. 7c that these areas correspond to the positive and negative footpoints of the AFS, named A and B, respectively (see also Fig. 2b). We corrected the magnetograms for the underestimation of the flux density following the results of Berger \& Lites (2003) and computed the emerged flux inside the selected areas: it is $3.03 \times 10^{20} \pm 5.16 \times 10^{19} \mathrm{Mx}$ and $1.56 \times 10^{21} \pm 2.17 \times 10^{20} \mathrm{Mx}$, for the positive and negative polarity, respectively. We estimated the errors in the calculated flux taking into account that the uncertainty in the MDI magnetograms is $\sim 15 \mathrm{G}$ (Zayer et al. 1996).

The above values of emerged flux correspond to a rate of emergence of about $2.49 \times 10^{19} \mathrm{Mx} / \mathrm{h}$ and $1.29 \times 10^{20} \mathrm{Mx} / \mathrm{h}$ for the positive and negative polarities, respectively. The five-toone imbalance between the two polarities cannot be explained in terms of the estimated errors and is probably due to the fact that the emerged positive flux is more diffused than the negative one, so that part of the positive flux might not have been taken into account in the computation of the emerging flux over the chosen threshold.

Moreover, to study the subsequent variations of the magnetic topology of the active region at the coronal level, we applied an extrapolation code based on a method introduced by Alissandrakis (1981). This method assumes that the magnetic field is force-free not only in the corona but also in the lower levels and that it vanishes at infinity. The magnetic field extrapolation was performed on magnetograms of $116 \times 116$ pixels $(232 \times 232 \mathrm{Mm})$. To minimize the oscillatory behavior of the Fourier solutions, we embedded the original arrays of the data in larger arrays $(232 \times 232$ pixels $)$ of the same resolution but with zeros up to the boundaries, before applying the 

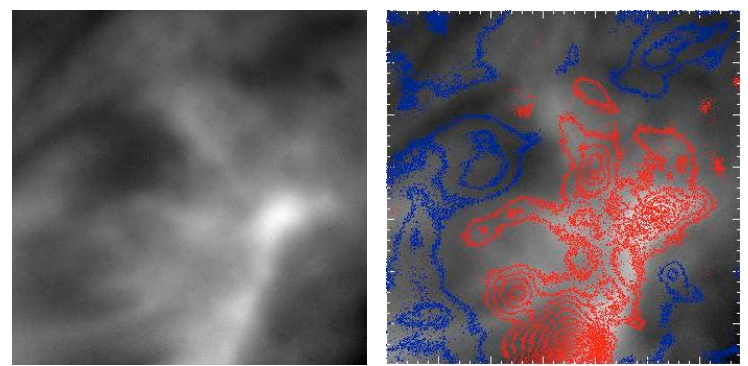

12:38 UT

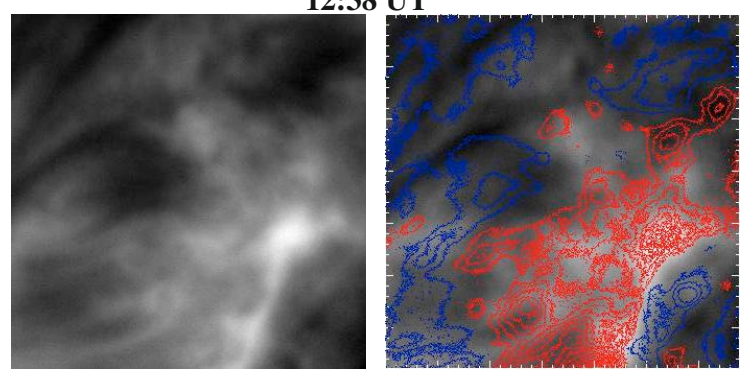

12:40 UT

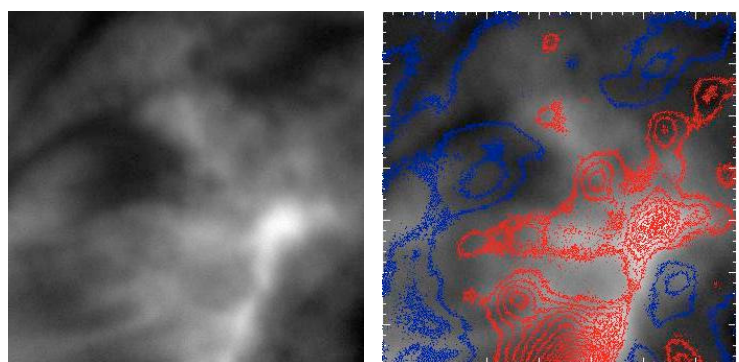

12:39 UT

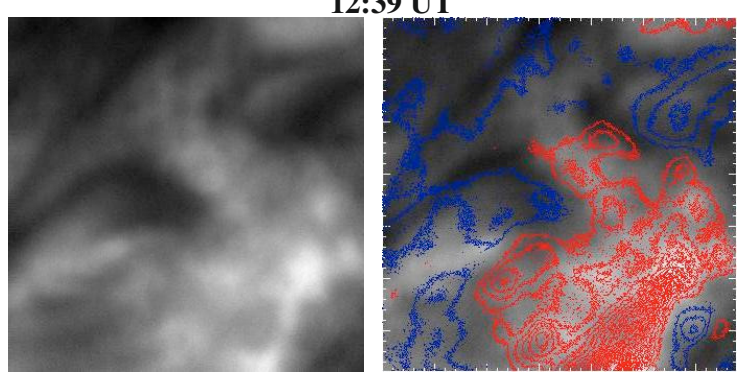

12:43 UT

Fig. 5. Selected sequence of images relevant to the field of view 3, acquired during the main phase but after the peak of the flare. Each pair of images shows the site brightness variation on the left and the associated velocity fields on the right. Blue (red) isocontours indicate upward (downward) velocity fields. The velocity contours are drawn every $1 \mathrm{~km} \mathrm{~s}^{-1}$. The fov is $24 \times 24 \mathrm{Mm}^{2}$.
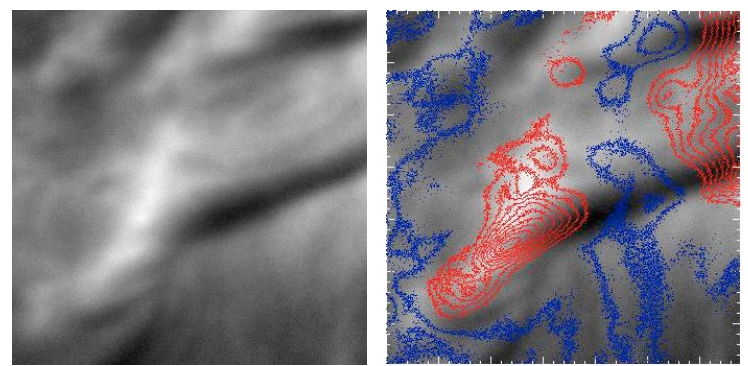

14:48 UT
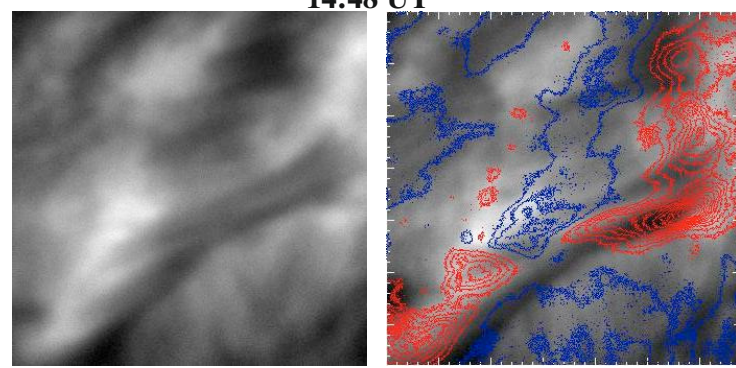

15:32 UT
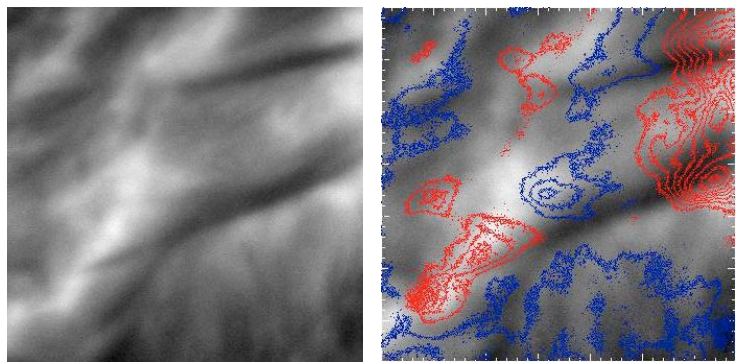

15:10 UT

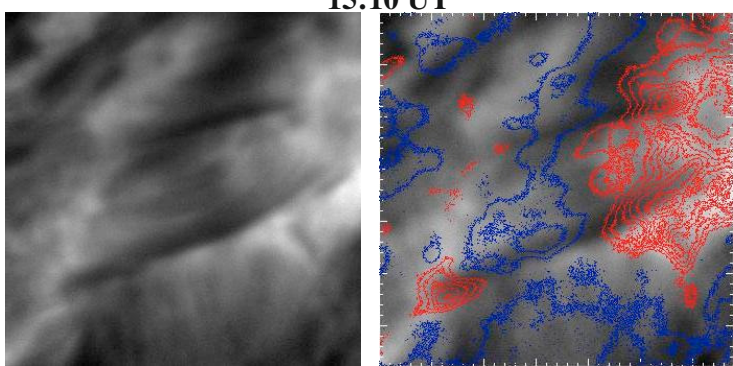

15:54 UT

Fig. 6. Selected sequence of images relevant to the field of view 4, showing the evolution of the AFS appeared between the two main spots (left) and corresponding velocity maps (right): blue (red) isocontours indicate upward (downward) velocity fields. The velocity contours are drawn every $1 \mathrm{~km} \mathrm{~s}^{-1}$. The fov is $24 \times 24 \mathrm{Mm}^{2}$.

extrapolation procedure. Figures 8a and 8c show the magnetic field extrapolations performed on the magnetograms taken on July 13 at 01:38 UT and at 12:14 UT. For both extrapolations we have taken a value of the $\alpha$ force-free parameter equal to $1.15 \times 10^{-2} \mathrm{Mm}^{-1}$, small enough to reduce the periodicity effects inherent to the Alissandrakis method and such as to provide a better fit of the computed field lines with the coronal loops observed by TRACE at the same time (Figs. $8 \mathrm{~b}$ and d). The most evident variation occurring in the coronal field before the beginning of the flare is the appearance of small loops, indicated by red lines in Fig. 8c, corresponding to the AFS observed in $\mathrm{H} \alpha$ (see Fig. 2b) and to the loop system at $171 \AA$ (indicated by the arrow in Fig. 8d).
To evaluate the relative inclination of the new field lines (red lines in Fig. 8c) with respect to the pre-existing ones (black lines in Fig. 8c), we have calculated the azimuthal angle $\gamma$ of the EUV loops in TRACE $171 \AA$ images. The azimuthal angle $\gamma$ is defined as the angle between the footpoint baseline and the circle of latitude parallel to the equator and passing through the eastern footpoints. We have used the IDL procedure Divergingloop (Contarino et al. 2006), based on the method developed by Loughhead et al. (1983) and on the assumptions that the loop footpoints can be determined, the loop central axis lies in a plane and the loop is symmetrical about an axis perpendicular to its baseline. We have found a relative inclination of $\sim 12^{\circ}$ between 


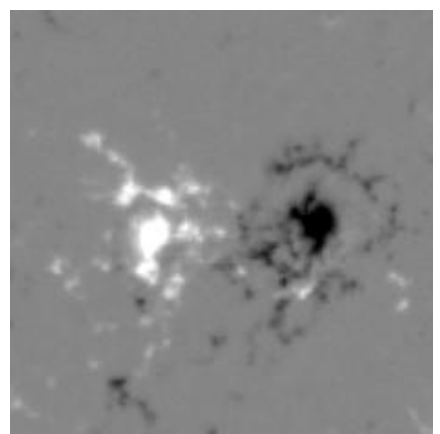

(a)

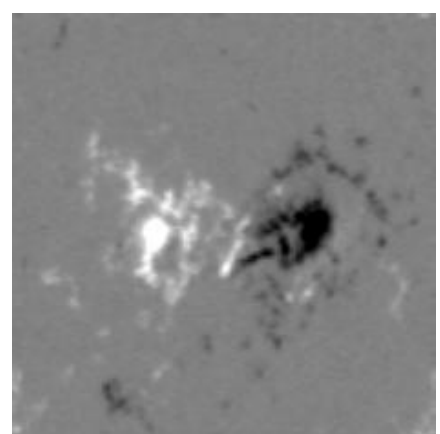

(b)

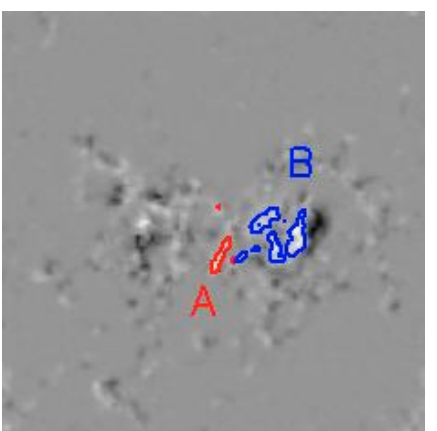

(c)

Fig. 7. a), b) Magnetograms of NOAA 10401 acquired on July 13 by SOHO/MDI in low resolution mode at a) 00:02 UT and b) 12:14 UT. The fov is $163 \times 163 \mathrm{Mm}^{2}$. c) Map difference between the magnetograms taken at 00:02 UT and at 12:14 UT. The white and dark regions indicate the areas of emerging and disappearing flux, respectively. The red and the blue contours indicate the regions characterized by an emerged flux greater than $2.6 \times 10^{18} \mathrm{Mx} /$ pixel. These regions correspond to the location of the positive (A) and negative (B) footpoints of the AFS.

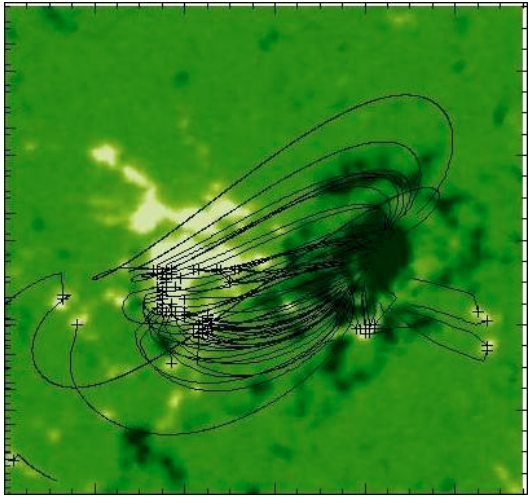

(a) 01:38 UT

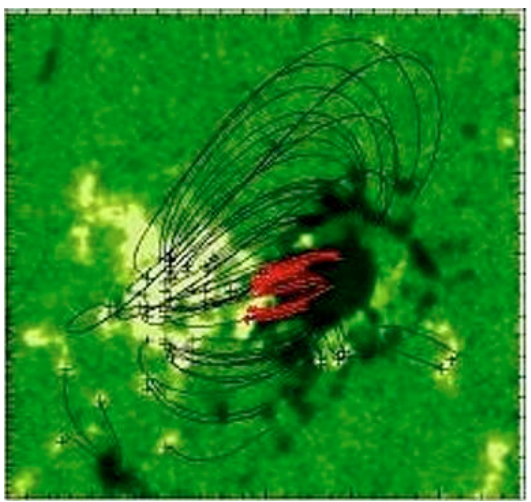

(c) 12:14 UT

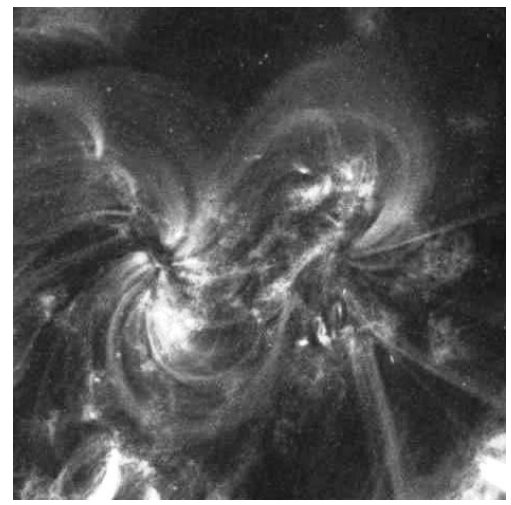

(b) 01:45 UT

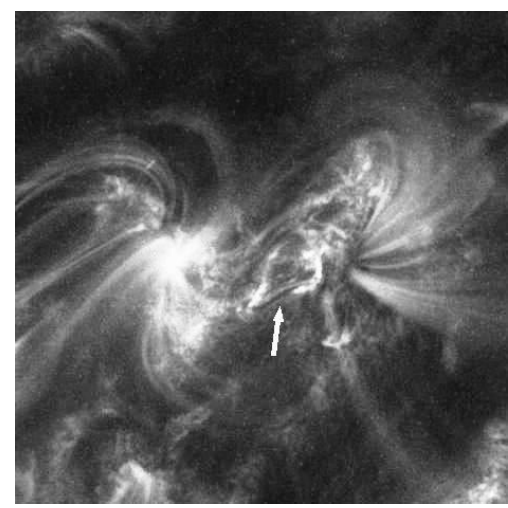

(d) 12:14 UT
Fig. 8. Magnetic field extrapolations of NOAA 10401 calculated using MDI magnetograms a), c), and coronal images taken by TRACE at $171 \AA \mathbf{b}$ ), d). In the magnetic field extrapolation obtained by the magnetogram acquired at 12:14 UT c), the red lines indicate the field lines connecting the red and blue areas evidenced in Fig. 7c. In the corresponding TRACE image d), these field lines are indicated by an arrow. Note that in the TRACE image acquired at 01:45 UT these field lines do not appear. The field of view is $232 \times 232 \mathrm{Mm}^{2}$. the two field line bundles, indicating that the two flux systems are nearly parallel.

An analogous result $\left(\sim 11^{\circ}\right)$ was obtained measuring the angle between the segment connecting the centroids of the two pre-existing polarities and the one connecting the centroids of the emerged areas A and B.

\section{Discussion and conclusions}

The C-level flare observed on July 13, 2003 occurred in a well developed, non-recurrent active region (NOOA 10401) that was present on the solar disk for 12 days, from its appearance at the East limb on July 6 until it disappeared at the West limb.

We notice the emergence of new magnetic flux within this active region on July 13 , specifically in the central area between the two main spots where several new pores can be noted before the flare occurrence (see, for instance, the TRACE WL image acquired on this day). This emergence is also indicated by a nonnegligible increase in the magnetic flux $\left(\sim 10^{21} \mathrm{Mx}\right.$, on average) within the considered area, where the AFS detected in $\mathrm{H}_{\alpha}$ data is located between the two main sunspots.

The dynamic behaviour of the AFS is similar to that observed in previous investigations (Spadaro et al. 2004; Zuccarello et al. 2005), both at the top and in the footpoints of the magnetic flux tubes, thus confirming the typical phenomenology observed in solar regions where new magnetic flux is emerging. The upward and downward plasma velocity values are consistent with those measured by the above authors, with the only exception of the AFS footpoints involved in the flare process, where the downward velocity is about a factor of 
two higher than is usually found. Moreover, our $\mathrm{H}_{\alpha}$ observations show that the AFS is continuing to rise within the area between the two main sunspots, even after the flare occurred.

For the downward velocity detected in the AFS footpoints involved in the flare process, we note, however, that it is about one order of magnitude lower than the values predicted by Galsgaard et al. (2007) for the outflows ejected sideways from the reconnection sites in the case of effective reconnection inside antiparallel flux systems.

Therefore, also taking into account that the relevant $\mathrm{H}_{\alpha}$ data where collected about 10 min after the flare peak, we argue that we are probably observing some remnants of the plasma flows caused by a weak reconnection process due to the interaction between the new emerging magnetic flux system and the preexisting field lines in the long-lived active region that might have given rise to the low energy $\mathrm{C}$-level flare. This hypothesis is in broad agreement with numerical simulations predicting very limited reconnection when the two flux systems have an almost parallel orientation (Galsgaard et al. 2007). The analysis of the loops observed in the $171 \AA$ images indicates that the relative inclination of the new field lines in the emerging AFS with respect to the pre-existing ones is only of $\sim 12^{\circ}$.

There is also some apparent disagreement between our results and the analysis of Galsgaard et al. (2007). They infer that, when the two flux systems are nearly aligned as in our case, it is possible for the emerging flux to penetrate into the existing coronal field before it creates any coronal signature revealing the process. In our case, instead, the initial weak reconnection appears to take place in the lower atmospheric layers, with higher plasma density. This is suggested by the fact that the peak in the EUV flare emission originating in the solar transition region occurred about 6 min earlier than the corresponding peak in the soft X-ray emission, produced after the energy released during the magnetic reconnection has propagated into the higher temperature coronal layers.

Moreover, we cannot rule out the alternative hypothesis that the flare was at the low end of the GOES scale because it involved a modest amount of magnetic flux: the emerged flux is $\sim 10^{21} \mathrm{Mx}$. Actually, the reconnection case studied by Longcope et al. (2005) showed that the emergence of about ten times more flux produced only a C-level GOES enhancement (peak flux 4-5 times higher than the pre-event background) lasting for about 5 times longer than the event reported in this work.

Therefore, it is important to extend numerical investigations of the interactions between initially unconnected magnetic flux systems, as well as to carry out high resolution observations directly tracking magnetic field lines, in order to confirm whether small class flares such as that detected in the AFS examined here are the result of reconnection processes occurring between the new emerging magnetic flux tubes and those pre-existing in the active region.

Acknowledgements. We wish to thank the THEMIS staff for their work during the observations, in particular the acting telescope operator and the support astronomer. Thanks are also due to L. Vlahos for providing the code used to extrapolate the force-free magnetic field. We thank the anonymous referee for her/his comments and suggestions which led to a sounder version of the manuscript. This work was supported in part by the Italian Ministry of University and Research, in part by the Istituto Nazionale di Astrofisica (INAF), in part by the Catania University, in part by the Agenzia Spaziale Italiana (contract I/035/05/0) and in part by the European Commission through the SOLAIRE Network (MRTN-CT-2006-035484).

\section{References}

Alissandrakis, C. E. 1981, A\&A, 100, 197

Berger, T. E., \& Lites, B. W. 2003, Sol. Phys., 213, 213

Contarino, L., Romano, P., \& Zuccarello, F. 2006, Mem. S. A. It Suppl., 9, 94

Galsgaard, D., Archontis, V., Moreno-Insertis, F., \& Hood, A. W. 2007, ApJ, 666,516

Longcope, D. W., McKenzie, D. E., Cirtain, J., \& Scott, J. 2005, ApJ, 630, 596 Loughhead, R. E., Wang, J. L., \& Blows, G. 1983, ApJ, 274, 883

Spadaro, D., Lanzafame, A. C., Consoli, L., et al. 2000, A\&A, 359, 716

Spadaro, D., Billotta, S., Contarino, L., Romano, P., \& Zuccarello, F. 2004, A\&A, 425, 309

Strous, L. H., \& Zwaan, C. 1999, ApJ, 527, 435

Zayer, I., Morrison, M., Tarbell, T. D., et al. 1996, A\&AS, 188, 3712

Zuccarello, F., Battiato, V., Contarino, L., et al. 2005, A\&A, 442, 661 